\title{
It's just not that easy! Challenges faced by nurses and midwives in the work environment in adhering to social distancing during COVID-19
}

\author{
AUTHORS \\ CASSANDRA HOBBS ${ }^{1,2}$ \\ LORNA MOXHAM $3,4,5$ \\ HEIDI GREEN ${ }^{2}$ \\ ELHAM ALMASI ${ }^{2,6}$ \\ REBEKKAH MIDDLETON ${ }^{3,7}$ \\ ELIZABETH HALCOMB 3,5 \\ RITIN FERNANDEZ ${ }^{1,2,3,5}$
}

1 St George Hospital, Kogarah, South Eastern Sydney Local Health District, New South Wales, Australia.

2 South Eastern Sydney Local Health District, New South Wales, Australia.

3 School of Nursing, Faculty of Science, Medicine and Health, University of Wollongong, Wollongong, New South Wales, Australia.

4 Global Challenges Program, Research and Innovation Division, University of Wollongong, Wollongong, New South Wales, Australia.

5 Illawarra Health and Medical Research Institute (IHMRI), University of Wollongong, Wollongong, New South Wales, Australia.

6 Sutherland Hospital, Caringbah, South Eastern Sydney local health District, New South Wales, Australia

7. Illawarra Health and Medical Research Institute, University of Wollongong, Wollongong, New South Wales, Australia

\section{CORRESPONDING AUTHOR}

CASSANDRA HOBBS St George Hospital, South Eastern Sydney Local Health District, Sydney NSW Australia. Mobile: +61 448375 708. Email: cassandra.hobbs@health.nsw.gov.au

\section{ABSTRACT}

Aim: The aim of this study was to understand the challenges that nurses and midwives face when seeking to practice social distancing within the various clinical settings in a hospital work environment during COVID-19.

Background: COVID-19 has had a significant impact on nurses and midwives internationally. With the ease of transmission of COVID-19 and the limitations in pharmaceutical interventions, other measures had to be implemented across communities and in healthcare settings. These public health measures were enacted in various ways throughout the world. A key measure employed globally was social distancing. Australia was no different, initiating community wide interventions to apply social distancing principles and action, in an effort to reduce transmission. Whilst at their places of work, nurses and midwives were also encouraged to practice social distancing.

Design and methods: Using convenience sampling, 579 nurses and midwives employed within one local health district in NSW, Australia completed an online questionnaire during the COVID-19 pandemic. Subsequently, a thematic analysis was undertaken as a way of categorising data from the 216 (37\%) qualitative responses with regards to social distancing. This study adhered to The Standards for Reporting Qualitative Research (SRQR) guidelines. ${ }^{1}$ 
Results: Overwhelmingly, responses indicated that participants found it challenging to practice social distancing in their workplaces across various clinical settings within the hospital. The two major themes identified were: 1 ) challenges relating to social distancing with patients and 2) challenges related to social distancing with colleagues. Several sub themes were also identified.

Conclusion: The COVID-19 pandemic has highlighted a number of challenges for healthcare professionals, social distancing being a key challenge. Social distancing is argued to be almost impossible in various clinical settings within a hospital where patient contact and provision of care in a team environment occurs.

\section{What is already known about the topic?}

- Social distancing has been demonstrated as an effective public health intervention to prevent the spread of infectious diseases, such as MERS, SARS and Ebola.

What this paper adds:

- During COVID-19, social distancing practices in acute healthcare environments have been enforced. However, this creates difficulties for health professionals such as nurses and midwives when providing quality care.

- The results showed that social distancing has been more difficult than anticipated to adhere to in the work environment.

- The built environment of hospitals (physical layout) and working in multidisciplinary teams made social distancing particularly challenging for nurses and midwives.

Keywords: Nursing, Midwifery, Acute care, Nursepatient relationship, COVID-19, Social Distancing.

\section{INTRODUCTION}

The World Health Organization (WHO) designated 2020 as the "Year of the Nurse and Midwife" and what a year it has been with an incomparable and extraordinary public health emergency SARS-CoV-2, known as COVID-19. ${ }^{2}$ Worldwide, nurses and midwives have met the unparalleled challenges associated with being at the forefront of healthcare during this respiratory pandemic. ${ }^{3}$ Nurses and midwives found themselves deployed in unfamiliar clinical settings, undertaking many different and varied tasks spanning from mass screening and health education, to providing care for people with COVID-19. ${ }^{4}$ Additionally, nurse led research teams are building a future evidence base of what was effective and what was not, with regard to the pandemic. Significantly, during the chaos of the initial pandemic period, nurses and midwives have continued to provide care for people who were hospitalised for health reasons other than COVID-19. Nursing and midwifery care simply did not stop, and every patient continued to receive care from a professional cohort committed to better health outcomes.

\section{BACKGROUND}

Given the ease of transmission of COVID-19, numerous public health measures including social distancing were enacted by various governments around the world. Greenstone and Nigam state that the core aim of social distancing is to keep people apart to reduce the mixing of susceptible and infectious people through early discovery of cases or through the reduction of contact that each person makes. ${ }^{5-7}$ The effectiveness of social distancing was demonstrated during the $\mathrm{H}_{1} \mathrm{~N}_{1}$ influenza pandemic in 2009 where implementation contributed to considerable decline in transmission rate in workplaces. ${ }^{8}$ Therefore, in March 2020, with the COVID-19 pandemic straining healthcare resources worldwide, ${ }^{9}$ the Australian Government implemented community wide social distancing with encouragement to work at home where possible and a discontinuation of non-essential gatherings (including gyms, licenced clubs, cinemas, restaurants, places of workshop, weddings and funerals. ${ }^{10}$ In April 2020, restrictions were increased, and schools were closed. ${ }^{11}$

All hospitals in New South Wales (NSW) were also required to adhere to the containment measures. As per the NSW Health guidelines, the containment measures implemented by hospitals included (1) closure of the multiple entrances and having a single-entry point to the hospital, (2) screening, (3) droplets and contact precautions and (4) social distancing. Screening involved the use of Quick Response ( $\mathrm{QR}$ ) codes and temperature checking using infra-red thermometers at hospital entrances for all staff. Out-patient medical visits where possible were conducted using telehealth and only emergency surgery and procedures were carried out. In the wards, visitation was restricted to one person only and families were encouraged to use video and audio methods to communicate with patients.

Droplet and contact precautions included strict hand hygiene where staff were provided with education about the importance of hand hygiene and hand sanitisers were placed at convenient locations and on mobile workstations to improve compliance. Mandatory education was also provided to staff regarding donning and doffing and masks 
were required to be worn when attending patient care. Guidelines for the use of personal protective equipment (PPE), including facemasks and gloves, were also provided to all staff. However, limited availability of PPE early in the pandemic meant that social distancing was a key measure to reduce transmission of COVID-19. Social distancing was mandated therefore seating and tables were removed from the hospital cafeterias and the occupancy of staff tea rooms was restricted. Notices were placed on break out rooms that indicated number of people allowed in the rooms. All staff were advised to maintain 1.5 metres between individuals hence ward-based meetings involving multiple staff were conducted using the cloud-based video conferencing service Zoom. At the time of this study each hospital participating in the study had directives in place for all public health measures including social distancing. The hospital directives including those relating to social distancing were reinforced during live updates provided by the General Managers of the individual hospitals as well as the Chief Executive of the Local Health District (LHD).

Given the myriad of clinical interactions that occur in the hospital environment, adherence to social distancing can prove challenging for healthcare professionals. ${ }^{12}$ These measures are particularly challenging for nurses and midwives who continue to provide front line care during a public health crisis. ${ }^{4}$ In the hospital setting, nurses and midwives have significant close contact in their daily interactions with patients who may potentially be infectious. Consequently, the reality of social distancing practice in acute healthcare environments requiring nurses and midwives to care for people alongside colleagues creates enormous challenge. Therefore, it is vital to understand the experiences of nurses and midwives when seeking to practice social distancing within their places of employment. Specifically, the first voice is privileged as nurses and midwives identified what they perceived as the challenges from the lived experience of delivering nursing and midwifery care.

\section{AIM}

The aim of this study was to explore the challenges that nurses and midwives faced when seeking to practice social distancing in the various clinical settings within the hospitals in one LHD during the COVID-19 pandemic.

\section{METHODS}

\section{DESIGN}

This was an analysis of the responses to an open-ended question that was included within a larger cross-sectional study. The larger study sought to explore the impact of COVID-19 on nurses and midwives wellbeing in a LHD in NSW Australia. This paper reports on the qualitative data collected about the participants' experiences of social distancing in the workplace. Data about other aspects of the survey is reported elsewhere. ${ }^{13,14}$ This paper is reported according to the Standards for reporting qualitative research guidelines, ${ }^{1}$ thus improving the transparency of all aspects of the research.

\section{PARTICIPANTS}

The study participants were a convenience sample of 579 nurses and midwives from approximately 3,00o employed within one LHD in NSW, Australia during the COVID-19 pandemic.

\section{DATA COLLECTION}

Data were collected using a self-administered online survey that was distributed to participants via email. The email contained a SurveyMonkey link and was distributed via a hospital wide email to all nurses and midwives by the Director of Nursing and Midwifery at each participating hospital. The survey commenced with a participant information sheet detailing the aims of the study and how the data will be used. The survey was conducted between May and June 2020, which was during the period the Australian government had affected restrictions on nonessential movement (social distancing and quarantine) of the Australian population. Reminder emails were sent by the Director of Nursing to improve response rates.

This paper reports on an open-ended survey item that asked participants to comment on their experience with practicing social distancing within an acute care hospital. The question was "When it is not a matter of patient safety, do you think that it is difficult to practice social distancing in a hospital environment?" which asked participants to rate the question as very easy, easy, neutral, difficult and very difficult. The follow-up question for the qualitative response was "If difficult, can you please explain why it is difficult to practice social distancing?".

\section{DATA ANALYSIS}

Data were managed within a Microsoft Excel spreadsheet. A thematic analysis, using the approach described by Braun and Clark was undertaken as a way of categorising data from the 216 responses received..$^{15}$ The data underwent multiple readings to identify important patterns with each researcher undertaking this individually. This process generated open codes which were then grouped together and given a brief description to aid in identifying the preliminary themes. The coded extracts were consistently discussed within the research team until consensus was reached on the final themes and appropriate nomenclature. Data analysis culminated in two overarching themes. These were consequently named and defined according to their distinct and distinguishing features. 


\section{ETHICS}

Ethics approval was gained for the study from the LHD Human Research Ethics Committee (2020/ETH01075). Completion and submission of the survey was considered as implied consent. Participants were informed that the survey was anonymous and that responses they provided will be used in any future publications.

\section{RESULTS}

A total of 579 nurses and midwives from approximately 3,000 employed across the LHD completed the survey for the larger study. However, only 452 nurses and midwives rated their difficultly in practicing social distancing within the hospital environment. Of these, 216 nurses and midwives provided qualitative comments relating to the question about social distancing. The majority of the nurses were females $(n=182$; $84.2 \%$ ), with a mean age of 43 years (SD 12.21) and the length of time they worked as a nurse or midwife was 18.1 years (SD 12.5). Nurses and midwives worked across a diverse range of clinical units including medical and surgical wards, intensive care, aged care, outpatient settings and maternity units. (Table 1).

Data analysis revealed a total of 331 of the 452 nurses and midwives (73\%) indicated that it was difficult or very difficult to practice social distancing within the hospital environment. Two key themes emerged from the 216 nurses and midwives in relation to the challenges they faced when seeking to practice social distancing in the various clinical settings within a hospital environment. Both themes were informed by subthemes. The first theme, 'Challenges relating to social distancing with patients' has two sub-themes, 'patient care' and 'the nature of nursing'. The sub-theme 'patient care' was informed by procedures, tasks and nursing interventions, while subtheme 'the nature of nursing' was informed by close physical contact. The second theme to emerge from the data was 'challenges relating to social distancing with colleagues' and has three sub-themes, 'the built environment' referring to the physical space, 'people' which has been informed by too many people, and adherence and 'equipment' referring to use and access. These themes are represented in Figure 1.

\section{THEME 1: CHALLENGES RELATED TO SOCIAL DISTANCING WITH PATIENTS}

Nursing and midwifery are inherently hands-on professions and patient care requires nurses and midwives to be in close contact with the people for whom they provide care. This creates significant challenges when nurses and midwives seek to socially distance from their patients.
TABLE 1: DEMOGRAPHICS $(\mathrm{N}=216)$

\begin{tabular}{|c|c|c|}
\hline Demographic variables & Frequency & $\begin{array}{r}\text { Percentage } \\
\%\end{array}$ \\
\hline \multicolumn{3}{|l|}{ Gender* } \\
\hline Female & 182 & 84.2 \\
\hline Male & 22 & 10.2 \\
\hline \multicolumn{3}{|l|}{ Professional Designation* } \\
\hline Registered Nurse/Midwife & 89 & 41.2 \\
\hline Clinical Nurse/Midwife specialist & 30 & 13.9 \\
\hline Clinical Nurse/Midwife consultant & 24 & 11.1 \\
\hline Clinical Nurse/Midwife educator & 21 & 9.7 \\
\hline Nurse/Midwife Unit Manager & 16 & 7.4 \\
\hline Nurse/Midwife Manager & 12 & 5.6 \\
\hline $\begin{array}{l}\text { Others: Enrolled Nurse; Assistant in } \\
\text { Nursing; Nurse Practitioner }\end{array}$ & 2 & 0.01 \\
\hline \multicolumn{3}{|l|}{ Department/ Unit worked* } \\
\hline Surgical Ward & 25 & 11.6 \\
\hline Intensive Care Unit & 23 & 10.6 \\
\hline Medical Ward & 17 & 7.9 \\
\hline Outpatient Department & 13 & 6.0 \\
\hline Oncology & 10 & 4.6 \\
\hline Antenatal/Birthing/Postnatal & 10 & 4.6 \\
\hline Aged care & 7 & 3.2 \\
\hline \multirow{2}{*}{$\begin{array}{l}\text { Other: mental health; paediatrics; } \\
\text { operating theatre etc }\end{array}$} & 81 & 37.5 \\
\hline & $\begin{array}{r}\text { Mean } \\
\text { (years) }\end{array}$ & $\begin{array}{r}\text { SD } \\
\text { (years) }\end{array}$ \\
\hline Age & 43 & 12.21 \\
\hline Years working as nurse or midwife & 18.1 & 12.5 \\
\hline
\end{tabular}

* Some respondents did not complete all fields in the survey.

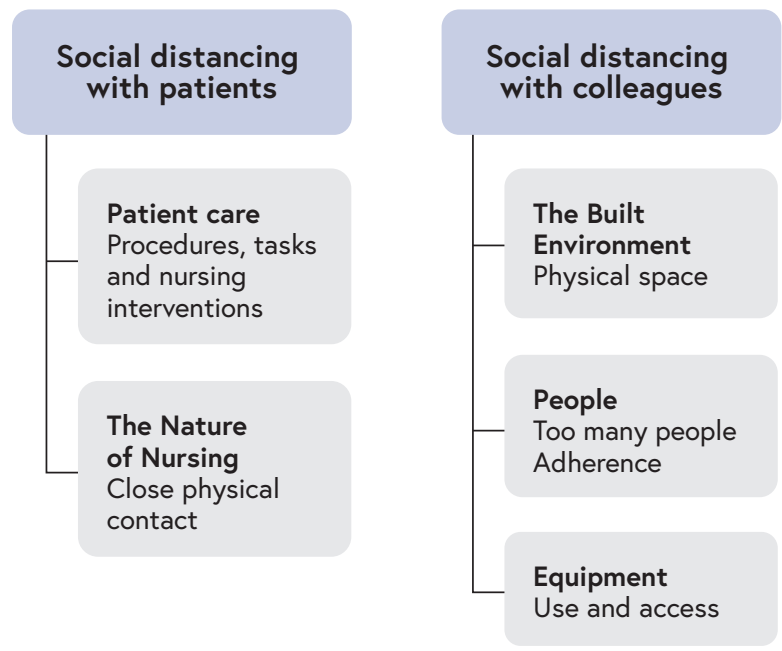

FIGURE 1: OVERVIEW OF MAIN THEMES 


\section{Sub-theme 1.1: Patient care}

The provision of patient care and the procedures, tasks and nursing interventions that is required for patient care could not occur if nurses and midwives had to remain $1.5 \mathrm{~m}$ apart from their patients. Comments regarding how impossible this was included, "Certain patient procedures require close contact e.g. line insertions, log rolls, doing ETT tapes, collar care just to name a few" (participant 27) and "You cannot treat them if you are 1.5 metres away from them e.g. Showering, dressings etc." (participant 206). Another participant described how social distancing in multiple situations such as, "During resuscitation, during dispensing of recordable drugs from Dangerous Drugs (DD) cupboard, administration and checking of medications which requires a two person check, and check at the bedside, handover at the bed side (trying not to share handover to other patients)" (participant 49), simply could not occur. Participants also responded to the question with their own rhetorical questions and statements such as: "How can you do any personal patient care from 1.5 metres?" (participant 58 ) and "It's very hard to deliver a baby from 1.5 metres away!" (participant 61).

\section{Sub-theme 1.2: The Nature of Caring}

The nature of caring sub-theme fundamentally encapsulates close physical contact. Participants expressed that "As nurses we need to be up close and personal with patients during some of their care" (participant 9) and that, "Showing empathy to patients and relations, requires physical touch" (participant 146). The "Nature of the job and workplace - our job requires you to get close to patients in order to fulfil your role" (participant 172). One participant summed this ethos up well when they said "Many interactions are physical, negating social distancing. The importance of touch is embedded in our practice of healing, making social distancing an anathema to our cultural practices" (participant 98). Yet another participant commented that "Care, comfort and empathy are difficult to achieve without touching” (participant 34).

\section{THEME 2: CHALLENGES RELATED TO SOCIAL DISTANCING WITH COLLEAGUES}

Just as patient care requires nurses and midwives to be in close contact with the people whom they provide care. Nursing and midwifery are often practiced in close contact and proximity to other nurses and midwives, other health professionals and ancillary staff. Participants described the challenges related to social distancing in the workplace as related to both the built environment and the nature of the work and interaction between people in the clinical setting.

\section{Sub-theme 2.1: The Built Environment}

Social distancing with colleagues was described by nurses and midwives as very challenging. The built environment was identified as an influencing factor. Nurses and midwives remarked that hospitals were not designed with physical distancing in mind. There was, quite simply "Not enough physical space to keep apart" (participant 38). Descriptions of the environment included observations such as "Narrow corridors, confined and shared workspaces" (participant 133), "Our unit is in a small cramped space" (participant 127), "Staff rooms, nurses station and wards are too small" (participant 160), and "A small area with a narrow corridor. The consultation rooms are also small, making social distancing difficult" (participant 171). Furthermore, as one participant explained, there was "Limited space at nursing stations. Limited computers. Ward meetings do not have the option for virtual meetings. Medication Room is a small space. Not all tearooms have enough space to safely practice social distancing" (participant 92). With the closure of the hospital cafeteria "it is difficult to find an area to sit at mealtimes and observe social distancing” (participant 53).

\section{Sub-theme 2.2: People}

In addition to the built environment, participants identified that people themselves made it challenging to adhere to social distancing. At times there were too many people in the clinical setting, who often did not or were unable to adhere to social distancing conventions. At times participants said the wards were quite crowded, with one saying "My ward has all the surgical specialities which means all the (specialist) teams end up on the ward at the same time with medical students. We also have nursing students and physio students" (participant 106). Another participant reported, "in ICU individual handover of patients occurs at each shift at one desk with four nurses present at a time" (participant 75). Another nurse spoke of the intensity of some critical clinical situations requiring many people to be present when they said, "I work in an ED. We cannot do our job while social distancing. A trauma patient may have $25^{+}$ people in the room working on them" (participant 69). Many participants felt that there was a lack of adherence to social distancing with one stating that "Some healthcare workers seem to ignore the advice and stand very close to each other" (participant 148). Others suggested that "Not everyone adheres to it" (participant 15), with "Some staff members not taking social distancing seriously and continue to enter rooms for chats and congregate for breaks" (participant 66). Two participants appeared to offer a rationale for the lack of social distancing between colleagues when they said: "We are by habit social creatures that are naturally drawn into each other's personal space" (participant 192) and "With such a low incidence rate, the general behaviour is business as usual" (participant 208). 


\section{Sub-theme 2.3: Equipment}

Use of and access to equipment was also identified as an issue that impacted nurses and midwives ability to socially distance. The participants described how there was not enough equipment and that it often had to be shared. One nurse said that the "Biggest issue is shared equipment and surfaces, e.g. computers, desk surfaces, physical patient notes, blood pressure devices etc." (participant 132). The availability of computers to use to access electronic medical records was also identified as posing a challenge because they were often only located in the nurse's station. One participant described how because of COVID-19, "In-service sessions (occurred) around a computer" (participant 151). This meant that nurse's stations were further crowded, for example, at the "Nurse's station we all sit close as computers are placed close together, and the offices are small" (participant 118). Another participant aptly illustrated this by saying "The area around the nurses station gets crowded, it's where all the phones and computers are. There's not enough space to implement social distancing" (participant 170).

\section{DISCUSSION}

In response to the COVID-19 pandemic, numerous public health measures, including social distancing, were implemented by healthcare facilities globally. This study was undertaken to provide insight into the experiences of nurses and midwives when seeking to practice social distancing in the various clinical settings within hospitals during COVID-19. The results of this study demonstrate the major challenges in implementing and maintaining social distancing in the various clinical settings within hospitals, and still engaging in patient care.

Social (physical) distancing in the various clinical settings within hospitals is critical to ensure the health and wellbeing of the health professional workforce during the pandemic. ${ }^{12}$ Protecting nurses and midwives, so that they can continue to care for patients, within an already stressed healthcare system, and without the fear of transmitting the virus, is crucial. ${ }^{8}$ As COVID-19 continues to spread rapidly across the globe, public health measures including social distancing are still required within hospitals. Hospital administration and policy makers need to urgently implement and provide employees with guidelines and strategies to address the challenges in social distancing within the workplace. Indeed, a study conducted by Courtemanche et al. ${ }^{16}$ demonstrated that the adoption of social distancing measures reduced the daily growth rate of confirmed COVID-19 cases in the US by $5.4 \%$ after one to five days, $6.8 \%$ after six to 10 days, $8.2 \%$ after 11 to 15 days, and 9.1\% after 16 to 20 days. While social distancing has been demonstrated to be effective within the community, in various clinical settings within a hospital this has been challenging for nurses and midwives during their daily roles. Adoption of new protocols during the height of the COVID-19 pandemic is vital in safeguarding nurses and midwives. To attain this, new behavioural expectations must be developed and reinforced surrounding social distancing including limiting traffic around the hospital and rearranging staff meal rooms.

While administrative meetings and education sessions have transitioned to online meeting platforms, patient care is unable to be performed in any way other than physical contact. Our study highlights that maintaining social distancing when providing patient care and having to share equipment is challenging, even impossible. Nurses and midwives reported that social distancing was impossible if effective care was to be provided. Touching and being close to patients to provide care is essential and part of developing and maintaining interpersonal relationships with patients and clients. The nature of clinical settings and hospital environments, including the physical layout of the workplace creates major barriers to practicing social distancing. Despite marked areas on floors for staff to stand during handover, and signage placed around workspaces stating how many staff could be present at any one time, providing care and adhering to social distancing was found to not be possible by participants in this study. This highlights the importance of access to appropriate PPE in the workplace when social distancing cannot be maintained.

\section{STRENGTHS AND LIMITATIONS}

This large study across three metropolitan hospitals offers a wealth of information on the challenges that nurses and midwives experience regarding social distancing measures in the various clinical settings. To our knowledge, at the time of writing, this is the first study to explore the impact of implementing public health measures such as social distancing on nurses and midwives within clinical settings in Australia. A limitation of this study is that the sample was drawn from a single local health district in NSW. However, this geographic region, which covers seven local government areas, has a complex mix of highly urbanised and industrialised areas and low-density suburbs and has a population of over 950,00o people. ${ }^{17}$ That said, a broader range of participants across other clinical settings would establish a more generalisable result. In addition, the data were drawn from a single qualitative item within a larger survey. More in-depth focus on social distancing may have elicited more detail. Additionally, the convenience sampling methodology used in this study could create bias, by under or over sampling the population. Further exploration of how social distancing can and does occur in clinical settings would be of benefit. Despite these limitations, the study was undertaken during the COVID-19 pandemic, providing real experiences of the impact of social distancing in practice for nurses and midwives. 


\section{CONCLUSION}

There are not many unseen benefits from the COVID-19 pandemic but nurses and midwives have demonstrated leadership and provided care not only to people affected by COVID-19, but also to other people who also require nursing treatment. Physical distancing is an important public health measure employed to slowing transmission of COVID-19. Clinical settings have not been immune to the need to adopt this concept. However, social distancing is not easy to adopt in the various clinical settings within a hospital environment. This study has identified challenges around the built environment, shared equipment and the presence of people that could be addressed to enhance nurses and midwives ability to practice social distancing. It is imperative for managers and policy makers to consider how these issues can be addressed to promote the safety of the workplace for staff and patients.

\section{IMPLICATIONS FOR RESEARCH, POLICY, AND PRACTICE}

The results of this study acknowledge the importance of social distancing as a preventative measure to limit transmission of COVID-19, however this study also reveals that social distancing in the various clinical settings within a hospital environment is difficult. This is especially if nurses and midwives are to continue to safely and adequately care for their patients. Further exploration of how social distancing can occur in clinical settings within hospital environments is needed for future pandemic planning and preparedness. Further research on how to optimise patient care while still adhering to social distancing measures, such as modification of handovers, and nursing and medical rounds is required.

Acknowledgements: The authors wish to thank the nurses and midwives who participated in this study.

Declarations of interest: The authors declare no conflicts of interest.

Funding Statement: This research did not receive any specific grant from funding agencies in the public, commercial, or not-for-profit sectors.

\section{REFERENCES}

1. O'Brien BC, Harris IB, Beckman TJ, Reed DA, Cook DA Standards for reporting qualitative research: a synthesis of recommendations. Acad Med 2014;89:1245-51.

2. World Health Organization. Year of the Nurse and the Midwife. World Health Organization. 2020. [cited 2020 Aug 26] Available from: https://www.who.int/campaigns/year-of-thenurse-and-the-midwife-2020

3. Jackson D, Bradbury-Jones C, Baptiste D, Gelling L, Morin $K$, Neville $S$, et al. Life in the pandemic: some reflections on nursing in the context of COVID-19. J Clin Nurs. 2020;0:1-3.
4. Fernandez R, Lord H, Halcomb E, Moxham L, Middleton R, Alananzeh I, et al. Implications for COVID-19: a systematic review of nurses' experiences of working in acute care hospital settings during a respiratory pandemic. Int J Nurs Stud. 2020:103637.

5. Payne J, Morgan A, Piquero AR. Covid-19 and social distancing measures in Queensland Australia are associated with shortterm decreases in recorded violent crime. Crime Sci. 2020;10:1-20

6. Greenstone M, Nigam V. Does social distancing matter? University of Chicago, Becker Friedman Institute for Economics. 2020. [cited 2020 Aug 26] Available from:

https://ssrn.com/abstract=3561244

7. Lewnard JA, Lo NC. Scientific and ethical basis for socialdistancing interventions against COVID-19. Lancet Infect Dis. 2020;20:631.

8. Prin M, Bartels K. Social distancing: implications for the operating room in the face of COVID-19. Can J Anaesth. 2020;67:789-97.

9. Kissler SM, Tedijanto C, Lipsitch M, Yonatan HG. Social distancing strategies for curbing the COVID-19 epidemic. medRxiv. 2020;2020.03.22.20041079

10. Australian Health Protection Principal Committee. Australian Health Protection Principal Committee (AHPPC) coronavirus (COVID-19) statement on 17 March 2020. Australian Government Department of Health. 2020. [cited 2020 Aug 26] Available from: https://www.health.gov.au/news/australianhealth-protection-principal-committee-ahppc-coronaviruscovid-19-statement-on-17-march-2020-0

11. Australian Health Protection Principal Committee. Australian Health Protection Principal Committee (AHPPC) coronavirus (COVID-19) statement on 22 March 2020. Australian Government Department of Health. 2020. [cited 2020 Aug 26] Available from: https://www.health.gov.au/news/australianhealth-protection-principal-committee-ahppc-coronaviruscovid-19-statement-on-22-march-2020-0

12. Arora VM, Chivu M, Schram A, Meltzer D. Implementing physical distancing in the hospital: a key strategy to prevent nosocomial transmission of COVID-19. J Hosp Med. 2020;15:290-1.

13. Fernandez R, Lord H, Moxham L, Middleton R, Halcomb E. Anxiety among Australian nurses during COVID-19. Collegian. 2021;28(4):357-8.

14. Middleton R, Loveday C, Hobbs C, Almasi E, Moxham L, Green $\mathrm{H}$, et al. The COVID-19 pandemic-A focus on Nurse Managers' mental health, coping behaviours and organisational commitment. Collegian 2021.

15. Braun V, Clarke V. Using thematic analysis in psychology. Qual Res Psych. 2006;3:77-101.

16. Courtemanche C, Garuccio J, Le A, Pinkston J, Yelowitz A. Strong social distancing measures In the United States reduced the COVID-19 growth rate: study evaluates the impact of social distancing measures on the growth rate of confirmed COVID-19 cases across the United States. Health Aff. 2020;39:1237-46.

17. NSW Health. South Eastern Sydney. NSW Government. 2020. [cited 2020 Aug 26] Available from: https://www.health.nsw.gov.au/lhd/Pages/seslhd.aspx 